\title{
Development of a Human Extracellular Matrix for Applications Related with Stem Cells and Tissue Engineering
}

\author{
Carmen Escobedo-Lucea • Angel Ayuso-Sacido • Chen Xiong • Sonia Prado-López • \\ Manuel Sanchez del Pino • Dario Melguizo • Carmen Bellver-Estellés • \\ Susana Gonzalez-Granero • M. Luz Valero • Rubén Moreno • Deborah J. Burks • \\ Miodrag Stojkovic
}

Published online: 28 June 2011

(C) The Author(s) 2011. This article is published with open access at Springerlink.com

\section{Introduction}

The recent progress in stem cell biology has created new approaches for their study as well as their application to the treatment of human diseases [1-3]. The success of stemcell based technologies in the clinical setting $[4,5]$ has emphasized the need to improve the standards of quality for all phases of cell therapy, particularly the development of culture methods that circumvent products of animal origin since these might provoke infections or immune rejection following transplantation in patients. Indeed, since Martin et al. (2005) [6] demonstrated that hESC cultured with

Deborah J. Burks and Miodrag Stojkovic contributed equally to the manuscript.

Electronic supplementary material The online version of this article (doi:10.1007/s12015-011-9270-6) contains supplementary material, which is available to authorized users.

C. Escobedo-Lucea $(\bowtie) \cdot$ S. Gonzalez-Granero

Comparative Neurobiology Unit, Instituto Cavanilles,

University of Valencia- RETICS,

46980 Valencia, Spain

e-mail: esluma@uv.es

C. Escobedo-Lucea $\cdot$ A. Ayuso-Sacido $\cdot$ C. Xiong $\cdot$

S. Prado-López • D. Melguizo • C. Bellver-Estellés · R. Moreno •

D. J. Burks $\cdot$ M. Stojkovic

Regenerative Medicine Program,

Centro de Investigación Principe Felipe,

46012 Valencia, Spain

M. S. del Pino $\cdot$ M. L. Valero

Proteomics Service, Centro de Investigación Principe Felipe,

46012 Valencia, Spain

D. J. Burks

CIBER of Diabetes and Metabolic Diseases,

Instituto de Salud Carlos III,

Valencia, Spain animal or serum products retained non-human sialic acid which was immunogenic when transplanted in humans, the establishment of animal-free conditions to support the maintenance and differentiation of human stem cells has been a major goal of the field of regenerative medicine [7]. Thus, chemically-defined culture systems that are devoid of non-human substances will greatly facilitate the use of stem cells in regenerative strategies.

The concept of a niche is crucial for the organization of stem cells. A niche is consider as a subset of tissue cells and extracellular substrates (matrix and soluble factors) that can support stem cells and control their self-renewal in vivo [8].

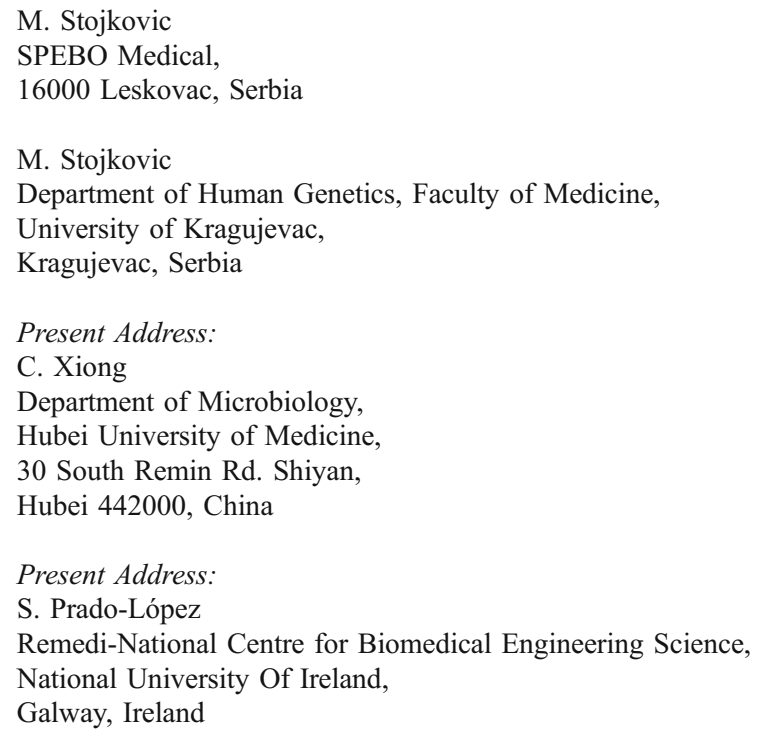


Extracellular matrices help to structure niches spatially and modulate the concentration of adhesive and signalling molecules locally. The ECM is a molecular complex that contains collagens and other glycoproteins, hyaluronic acid, proteoglycans, glycosaminoglycans (GAGs), and elastins. Additionally, the ECM harbours growth factors or cytokines to protect against degradation [9]. ECM components are responsible for adhesion during the majority of cell interactions and are implicated in the maintenance of embryonic induction during development as well as stem cell differentiation in vitro [10]. Thus, local changes in ECM can dramatically modulate the proliferation and migration of stem cells and may participate in the specification of lineages.

hESC have provided invaluable tools for gaining insight into the developmental origins of human tissues. However, to realize the full biological and clinical potential of hESC, certain problems related with the routine culture of these cells must be solved. Mouse embryonic fibroblasts (MEFs) and murine derivatives such as Matrigel are widely used in the maintenance and differentiation of hESC. Recently, considerable effort has been dedicated to the elimination of animalderived reagents from the culture of hESC and in parallel, to the control of cell growth parameters by avoiding human feeder cells. For example, in 2006 Ludwig et al. reported the use of conditioned media and high doses of FGF to maintain the undifferentiated state in hESC cultured on plastic, but some abnormalities were detected at passage 20 under these conditions [11, 12]. The use of human feeders complicates the growth and molecular analysis of both pluripotency and differentiation since experimental data may reflect the combined effects of hESC and feeder cells in the culture. Given these considerations, the use of an ECM of human or synthetic origin would provide many advantages. Indeed, there have been attempts $[13,14]$ to produce such a tool but the results have not been satisfactory because the products were unable to maintain hESC in the undifferentiated state over time. Matrix proteins have been used as coating for in vitro cultures of human stem cells but they have usually been applied as undefined protein mixtures [11] of animal origin [15] with undefined media [13], serum [16] or a synthetic mixture [17, 18]. However, most of these human biological reagents are expensive to manufacture and thus, are costprohibitive for many laboratories.

Decellularization procedures have been used traditionally to isolate ECM from cells in culture, tissues or organs [19]. The goal of decellularization protocols is to efficiently remove cellular and nuclear material while minimizing any adverse effect on the composition, biological activity and mechanical and structural integrity of the remaining ECM [20, 21]. Decellularized human scaffolds have facilitated the remodelling of various tissues in both animal models and humans [4, 22]. However, any biochemical procedure employed to remove cells may also alter the native three-dimensional architecture of the ECM and thus, a balance must be achieved between chemical and physical treatments during the decellularization process [4, 5, 23].

Given the potential importance of hESC in translational research and regenerative medicine, the aim of the present study was to develop a simple, efficient protocol for the production of a human ECM that is both safe and economical. Here we report that hypotonic lysis of human foreskin fibroblasts (HFF) generates a human ECM that retains protein components which are essential for attachment and cell-cell interaction. This hffECM was capable of maintaining the pluripotency of hESC and supporting their differentiation when used with the appropriate medium. Therefore, our results reveal hffECM as a novel tool which may facilitate the clinical application of hESC-based technologies.

\section{Results}

Optimisation of Conditions for the Generation of a Functional ECM from Human Foreskin Fibroblasts

To obtain a human ECM which would support the maintenance of undifferentiated stem cells, we initially designed a protocol using RIPA buffer to extract ECM from cultured HFF [24]. However, to optimise this matrix for clinical applications, we contemplated the use of a buffer to induce osmotic shock, thereby circumventing the use of detergents to lyse attached fibroblasts. Thus, HFF (passage 11-18) were grown to confluence and then inactivated with mitomycin C. Subsequently, these cultures were lysed with a Tris-Buffer which triggered osmotic shock. Incubation with this buffer caused the cells to swell and detach from the plate. This process was facilitated by six washes with PBS to remove broken cells and debris (see Fig. 1 for the details).

\section{Structural Characterization of hffECM}

To assess whether ECM proteins were retained after the cell lysis procedure, plates were examined by various microscopy techniques. Control plates of intact HFF (Fig. 2 a1-a4) and experimental cell-free plates (Fig. 2 a5-a8) were fixed and processed for ultrastructural analysis using transmission electron microscopy (TEM). The optimal length of cell culture for the obtention of hffECM was determined by assessing HFF which were cultured for either two, nine or 12 days prior to lysis (Fig. 2a). Following treatment with lysis buffer, intact fibroblasts were not observed on any of the plates as verified by DAPI staining of nuclei (Fig. 2 


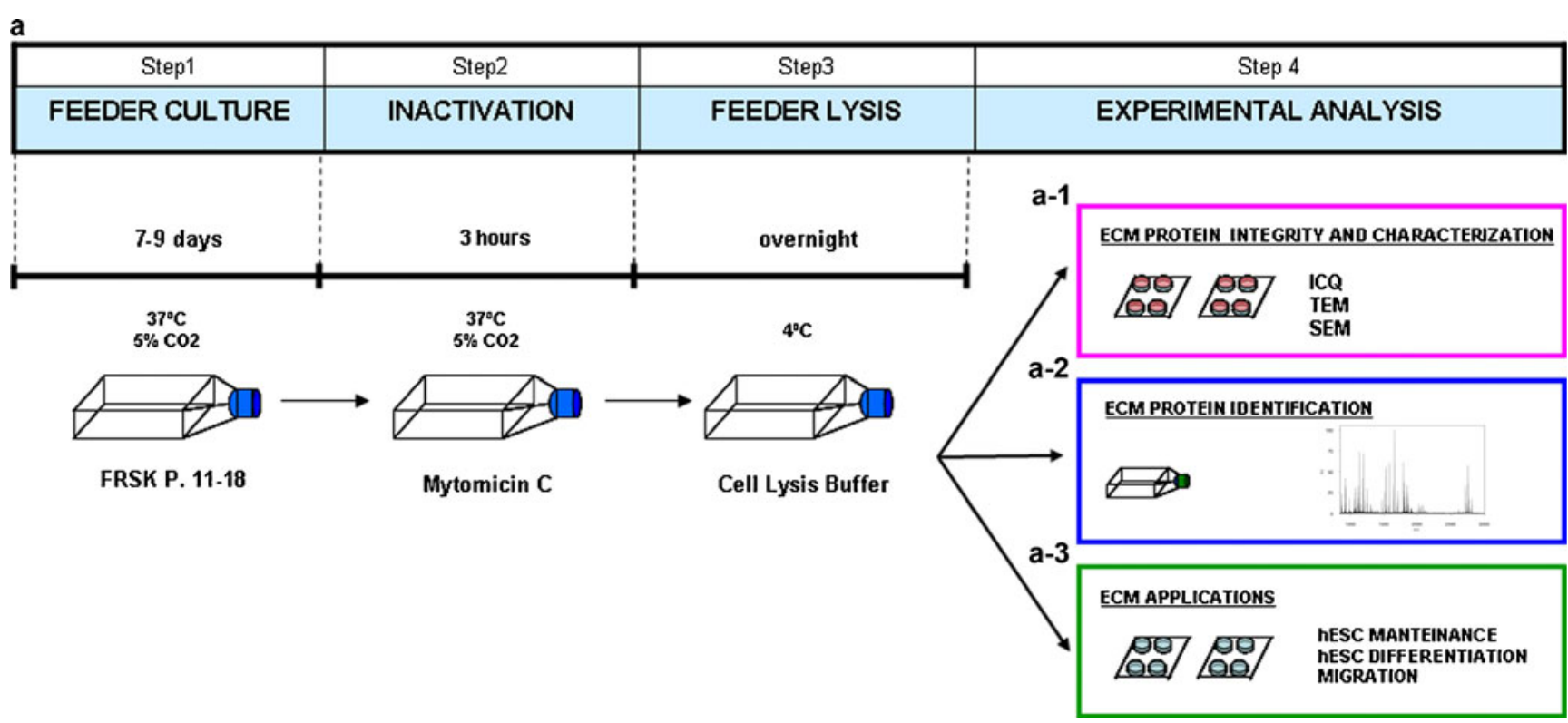

Fig. 1 Experimental design for the obtention, analysis and applications of hffECM. Human foreskin fibroblasts (passage 11 to 18) were cultured during 7-9 days before inactivation with mitomycin $\mathrm{C}$. At this stage, cultures were treated overnight at $4^{\circ} \mathrm{C}$ with lysis buffer to remove cells and the resulting hffECM was subjected to one of the following procedures: (a-1) Assessment of hECM protein integrity and characterization. Electron microscopy analysis was performed to evaluate the integrity and composition of hECM after the treatment and to establish the optimal period for obtention (see Fig. 2a).

b2,4,6). The structure and distribution of the hffECM were preserved based on microscopic analysis (Fig. 2 a5). The hffECM obtained from HFF cultured for only 2 days had a low content of structural proteins (Fig. 2 a-6), as evidenced by the loose lattice of fibers, suggesting that two days of culture is insufficient for preparation of a quality hffECM. After 12 days of culture, protein fibers displayed signs of degradation (Fig. 2 a-8). Thus, the quantity and architecture of the ECM from HFF cultured for 7-9 days (see Fig. 2 a-7) suggested that this amount of time might provide the best quality cell-free matrix. The presence of characteristic ECM adhesion proteins before and after the treatment was confirmed by immunostaining (Fig. 2b) using antibodies against fibronectin (Fig. 2 b1,2), collagen (Fig. 2 b3,4) and laminin (Fig. 2 b5,6). Collectively, the results of these studies demonstrate that the hffECM retains a structure similar to the ECM of intact HFF, suggesting that the lysis conditions do not damage significantly the organization of the matrix.

Detergent-Free Cell Lysis Preserves the Structure and Composition of the hffECM

To characterize the composition of the matrix obtained from the human fibroblasts, we eluted the hffECM from culture plates and performed a proteomic study. A total of 220
Immunocytochemistry (ICQ) was used to confirm visually the presence of characteristic ECM components (see Fig. 2b). (a-2) Identification of ECM proteins by proteomic analysis (Fig. 3). (a-3) Application of hffECM to biological processes. To test the functional capacity of the hffECM, we designed and performed different experiments related with pluripotency (Fig. 4a-e) and differentiation of hESC towards the three germ layers (Fig. 5 and Supplementary Fig. 1a-f) as well as the migration of adults cells (Fig. 6 a-c and Supplementary videos $1-3$ )

proteins were identified with a false discovery rates (FDRs) below 5\% (Table 1, Supplementary information). To evaluate the origin of the proteins, the enrichment of the cellular compartment terms of gene ontology was analyzed using Babelomics [25, 26] (Supplementary Fig. 1). At level 4 , only the terms extracellular matrix (GO:0031012) and cell surface components (GO:0009986) were significantly enriched $(p<0.01)$. The function of the proteins included in these terms are highly related as indicated by the network created by STRING analysis (Fig. 3a). These results demonstrate that many of the proteins responsible for the interaction between the cell surface and the ECM network are present in the hffECM. Although laminin was detected in the intact hffECM by immunocytochemistry, we were not able to identify it by mass spectrometry in samples of eluted hffECM. The most probable explanation for this discrepancy is the low recovery of laminin from the culture dishes due to its low solubility once assembled in the ECM.

To obtain further information regarding the functional aspects of the hffECM proteins, the enrichment of Kyoto Encyclopedia of Genes and Genomes (KEGG) pathways was also analyzed. Of the ten pathways significantly enriched $(p<0.01$, Supplementary information), three are related with focal adhesions and the cytoskeleton and play important roles in cell proliferation, differentiation, and survival. A part of the focal adhesion pathway is the 

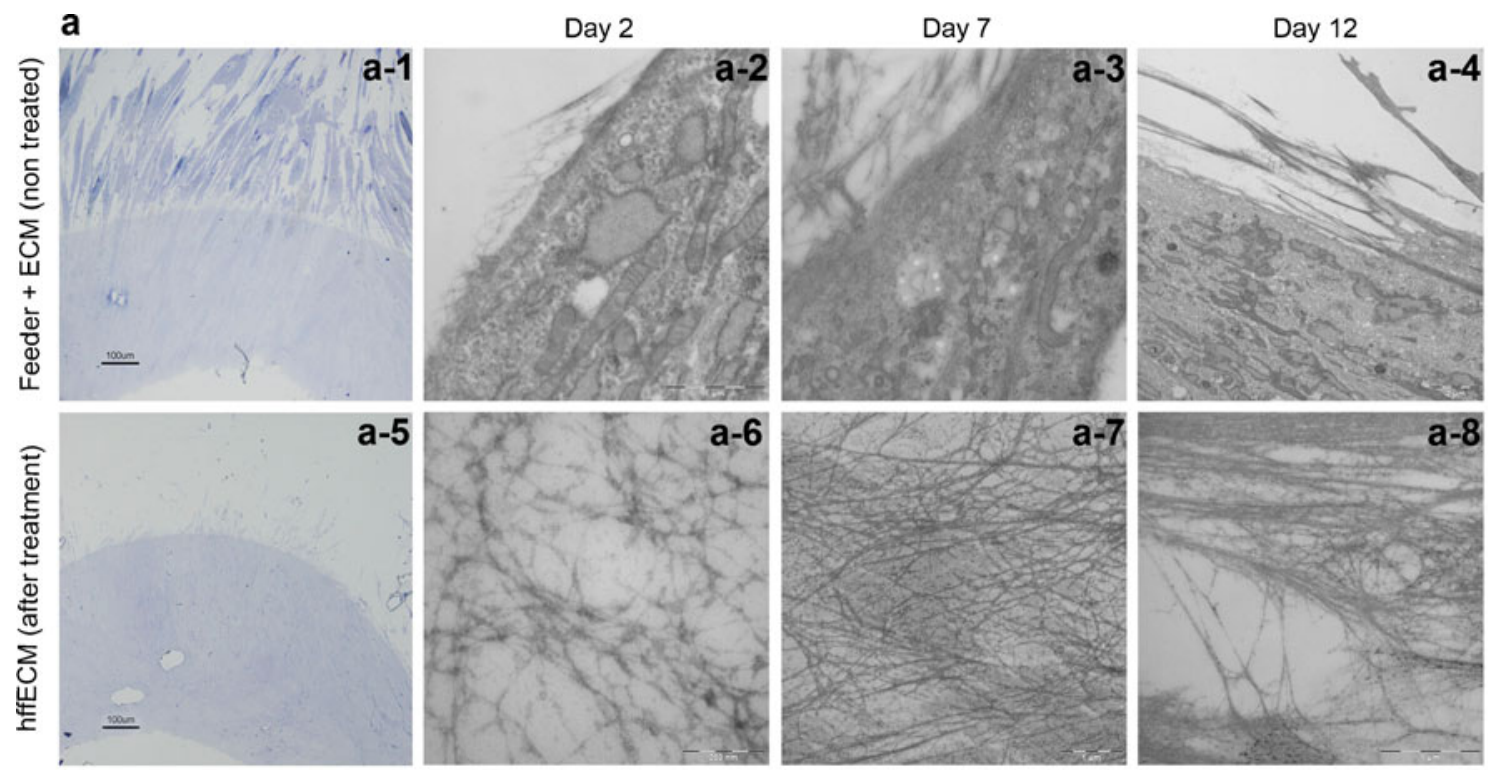

\section{b}

Non treated
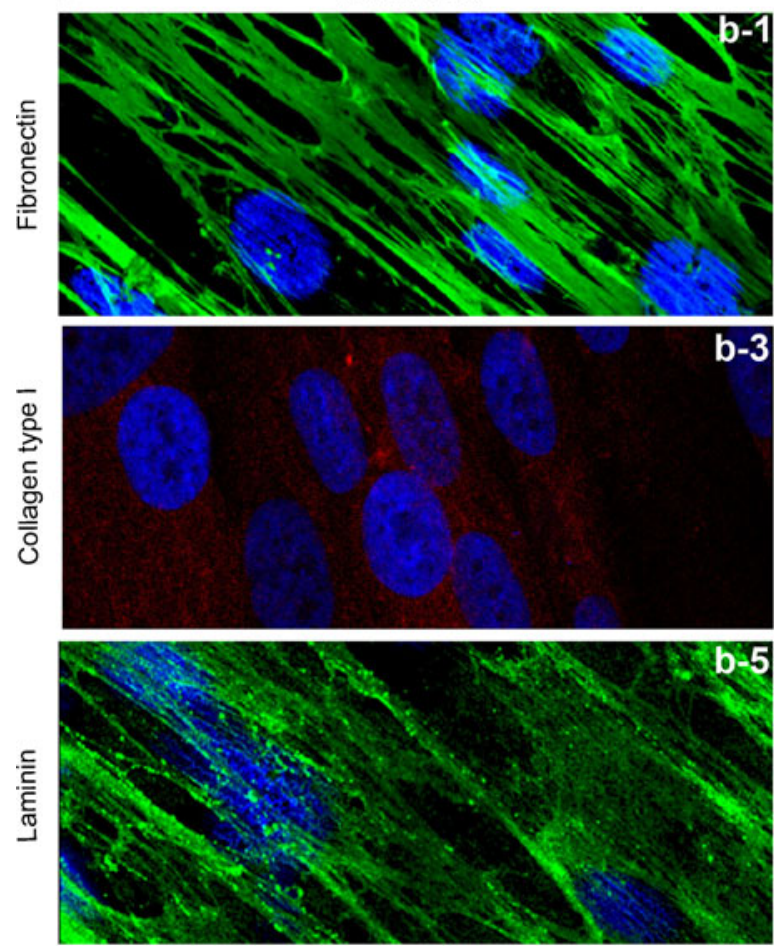

Fig. 2 Evaluation of the structural integrity of the hffECM. a Ultrastructural analysis of the efficiency of extraction protocol using transmission electron microscopy. Plates of lysed cultures and their respective controls with intact foreskin fibroblasts were fixed and processed for transmission electron microscopy ultrastructural analysis. To determine the optimal length of culture for production of hffECM, HFFs were maintained for the indicated times and then subjected to hypotonic lysis. (a1, a5) Semi-thin sections were prepared from control and lysed cultures and were stained with toluidine blue. (a2-a4) Representative TEM images of intact HFF control cultures at the indicated time-points. (a6-a8) Representative

extracellular matrix-receptor interaction pathway, which mediates the interactions with integrins and proteoglycans.
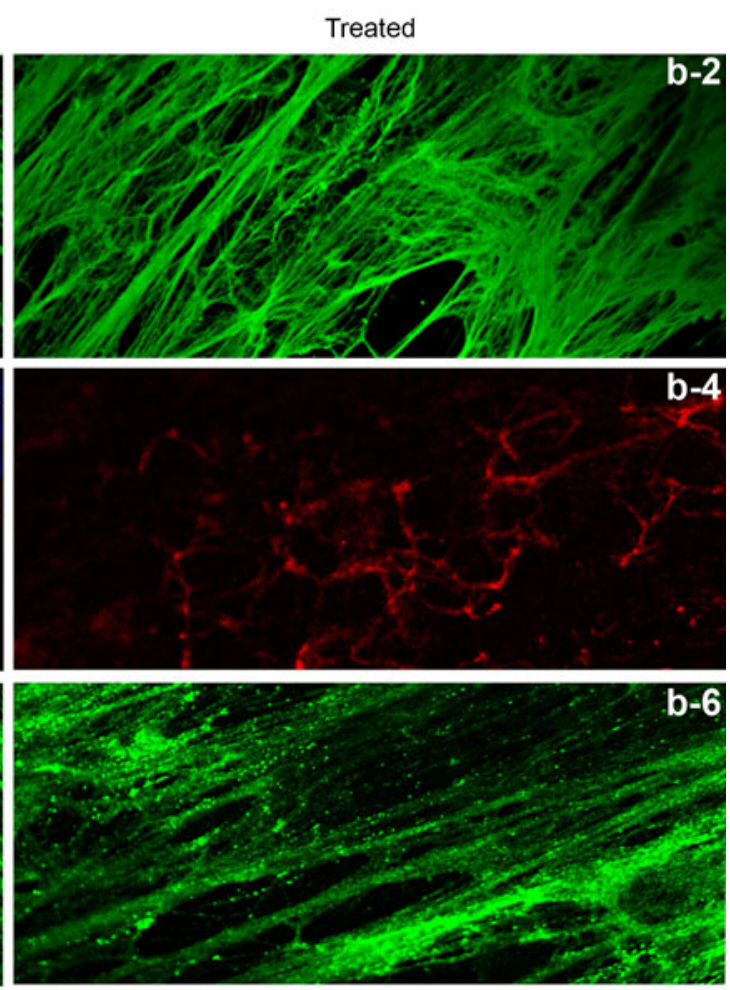

TEM images of lysed culture plates at the indicated time-points. Scale bars, $100 \mu \mathrm{m}$ (a-1 and a-5), $2 \mu \mathrm{m}$ (a-3 and $\mathbf{a}-\mathbf{4}), 1 \mu \mathrm{m}$ (a-2,a-7 and $\mathbf{a}-\mathbf{8})$. b Immunocytochemical analysis of structural proteins in hffECM. Control cultures of intact HFF or lysed plates were immunostained with antibodies against fibronectin (b-1, b-2), collagen type I (b-3, b-4) and laminin (b-5, b-6). Note that fibronectin, collagen type I and laminin were retained on plates after the treatment with lysis buffer. Cell nuclei as detected by DAPI were absent in lysed cultures. All images were captured at $63 \mathrm{X}$ magnification using a Leica confocal microscope, with the appropriate lasers to excite the secondary antibodies (Alexa, $488 \mathrm{~nm}$ ) and $405 \mathrm{~nm}$ for DAPI

As indicated in Fig. 3b, most of the extracellular partners of the pathway were detected in hffECM. Collectively, the 
Fig. 3 Identification of protein components of the hffECM.

After removal of HFF by lysis, the remaining hffECM was eluted from culture plates and subjected to proteomic analysis. a STRING analysis of the proteins contained in the cellular component terms of gene ontology that are significantly enriched in the hffECM samples. The two principal clusters correspond mainly to proteins of the extracellular matrix (blue) or cellular surface components (green). Note that the components form tight networks with several intercluster connections. b Protein components of the human ECMreceptor interaction pathway of the KEGG database. The proteins of the hffECM identified by mass spectrometry are in indicated in red. Laminin, in blue, was detected by immnunocytochemical analysis of the hffECM. Most of the extracellular matrix partners of the pathway have been identified a

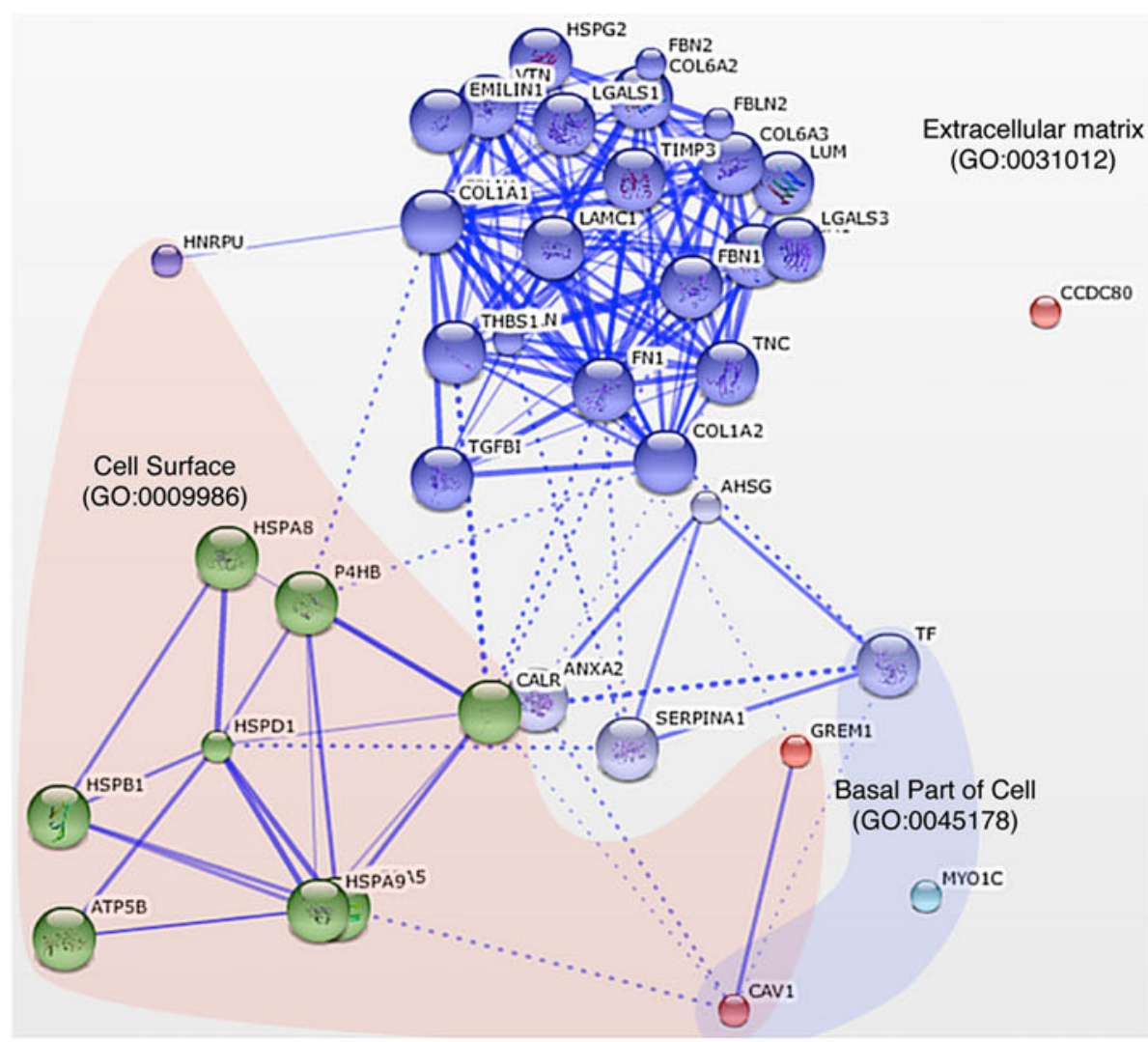

b ECM-RECEPTOR INTERACTION
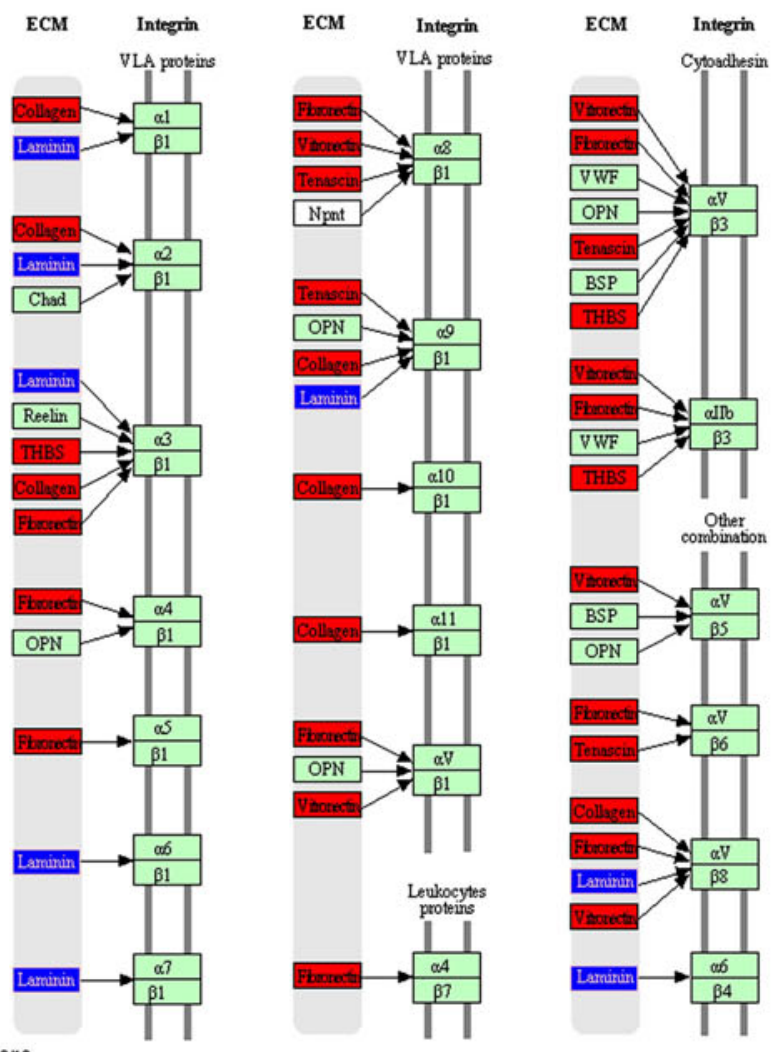

Focal adhesion

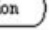

.

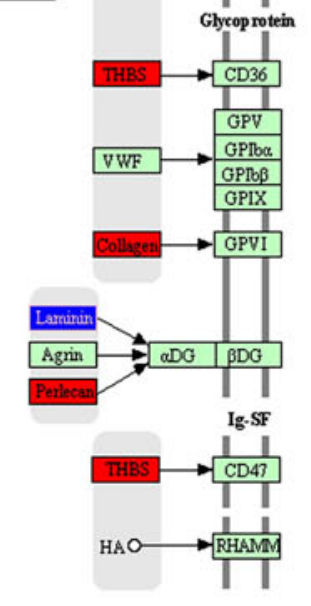


results of the proteomics analysis demonstrate that the procedure we have developed for preparation of hffECM retains most of the key components necessary for cell attachment and regulation of proliferation and differentiation. Regarding the other enriched pathways, some may reflect contaminants due to non-specific binding (e.g., ribosomal proteins and histones) whereas others may have been identified because they are anchored to the plasma membrane, cytoskeleton, and/or ECM. Interestingly, some glycolytic pathways were enriched (pathways hsa00620 and hsa00010). Although their origin is not clear, it is noteworthy that they are components of the anaerobic glycolysis pathway and one might speculate that they may be located at the extracellular space to provide energy locally to the ATP-dependent processes that may occur in this vicinity.

\section{hffECM Maintains Pluripotency of hESC}

Colonies of H9 (passage 31), maintained on HFF, were cut mechanically and distributed equally into 4 experimental groups. One group was again plated on HFF and maintained during the assay using traditional conditions for hESC culture. The other 3 groups of hESC colonies, were seeded onto hffECM-coated plates, using either TESR1 feeder-conditioned medium, feeder-conditioned medium 1 , or the traditional KSR medium $+\mathrm{bFGF}$ as a control. Subsequently, cultures were passaged mecanically every 5-6 days. Colonies seeded on human hffECM and maintained with KSR or conditioned medium 1 began to display a morphology consistent with spontaneous differentiation after 2 to 5 passages. In contrast, colonies plated on hffECM with TERS1 conditioned media retained the morphology of undifferentiated colonies with defined borders and a compact sphere, similar to hESC maintained on feeders (Fig. 4a, b). To ensure that colonies were indeed growing on the hffECM, cultures were stained with antifibronectin (Fig. 4b-3). Detection of this ECM component under and around the colonies suggests that the cells were plated on hffECM and not the plastic of the culture plate. DAPI was used to counter-stain the nuclei of cultured cells. In the image a-3 of Fig. 4, blue nuclei were observed in hESC colonies and feeder cells. However, in cultures maintained on hffECM, DAPI staining was detected exclusively in the colonies but not around them, reinforcing the observation that intact human feeder cells were eliminated during preparation of the hffECM. (For more details, see Supplementary Fig. 2)

After 21 passages on hffECM, the H9 colonies displayed a normal female kariotype of 46 XX (Fig. 4c). Telomerase activity was similar between H9 cells at the onset of the experiment (passage 31) and those maintained for 21 additional passages (total of 52 passages) on either hffECM or HFF (Fig. 4d). Additionally, the hESC maintained on hffECM continued to express markers of pluripotency at levels equivalent or superior to those detected in hESC grown on feeder layers (Fig. 4e). These data suggest that hffECM supports H9 colonies in the undifferentiated state. Similar observations were made with the $\mathrm{H} 1$ cell line.

\section{hffECM Supports Differentiation of hESC}

To facilitate clinical applications, ECM that meets the requirements of an animal-free product should also support differentiation of hESC. To test spontaneous differentiation, H9 colonies were allowed to overgrow on hffECM under conditions of bFGF deprivation for at least 2 weeks. Subsequent analysis by immunostaining revealed that the cultures had differentiated to ectoderm (Tuj1 positive cells) and mesoderm (cardiac actin) (Supplementary Fig. 3 a,b).

To determine the compatibility of the hffECM with the differentiation of hESC by chemically-defined protocols, H9 colonies were cultured on this matrix under conditions known to generate human adipocytes [27]. After 15 days in this differentiation medium (Fig. 5a), the cultures were analysed for lipid accumulation and markers of adipose tissue. We detected the presence of many Oil red O-stained lipid droplets in the cytoplasm of the cells on hffECM (Fig. 5b). Detailed ultrastructure analysis of differentiated cells using TEM revealed that their morphology was compatible with mature adipocytes (Fig. 5c), suggesting the functional capacity of this matrix to promote directed differentiation. To confirm that these cells were indeed adipocytes, the expression of adipose-specific genes was examined by RT-PCR using human adipose tissue as a positive control. Consistent with the presence of lipid droplets, aP2 and PPAR $\gamma$, transcriptional factors which are required for adipogenesis, were up-regulated significantly after 3 days in differentiation medium, demonstrating that the hffECM promotes differentiation of hESC under defined conditions. Consistent with this, when H9 grown on hffECM were subjected to a published protocol for the step-wise induction of endoderm [28], markers of definitive endoderm were observed after 3 weeks (Supplementary Fig. 3 c-f). Additionally, an earlier version of our hffECM was used successfully for differentiating H9 to endothelial precursors and mature endothelial cells [29].

\section{hffECM Supports Cell Migration in 3-D}

The removal of adhesion proteins and GAGs from the ECM would be expected to slow cell migration on the coated 


\section{a}

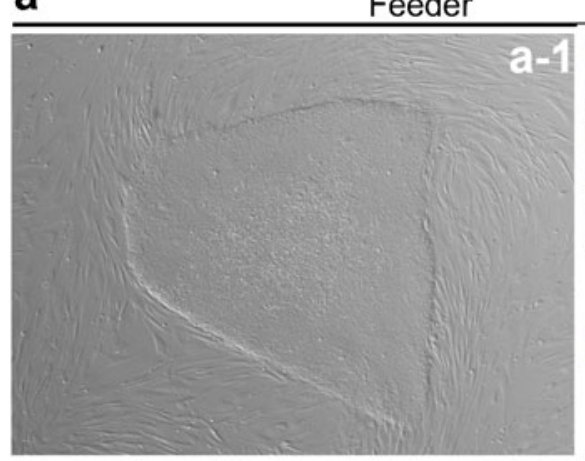

b
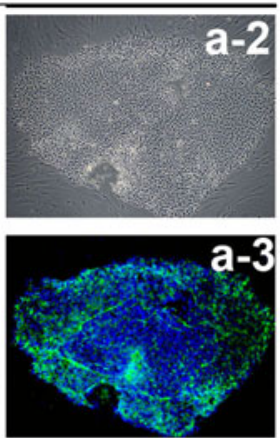

hffECM

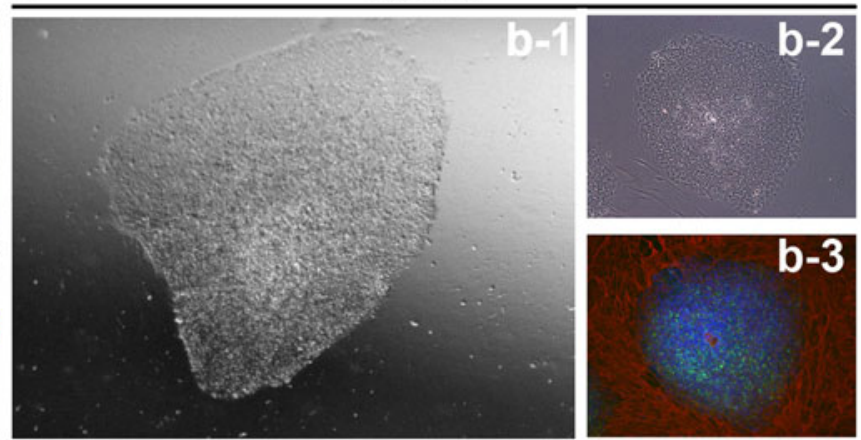

C

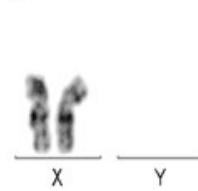

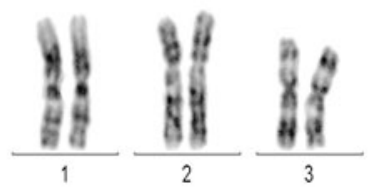
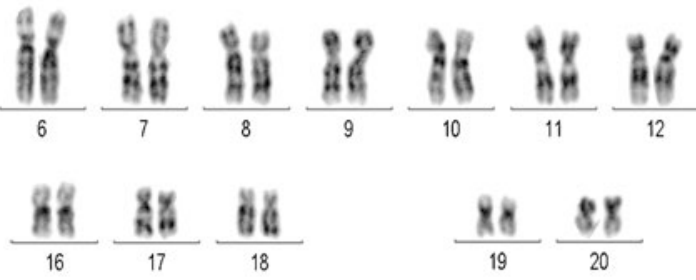

e

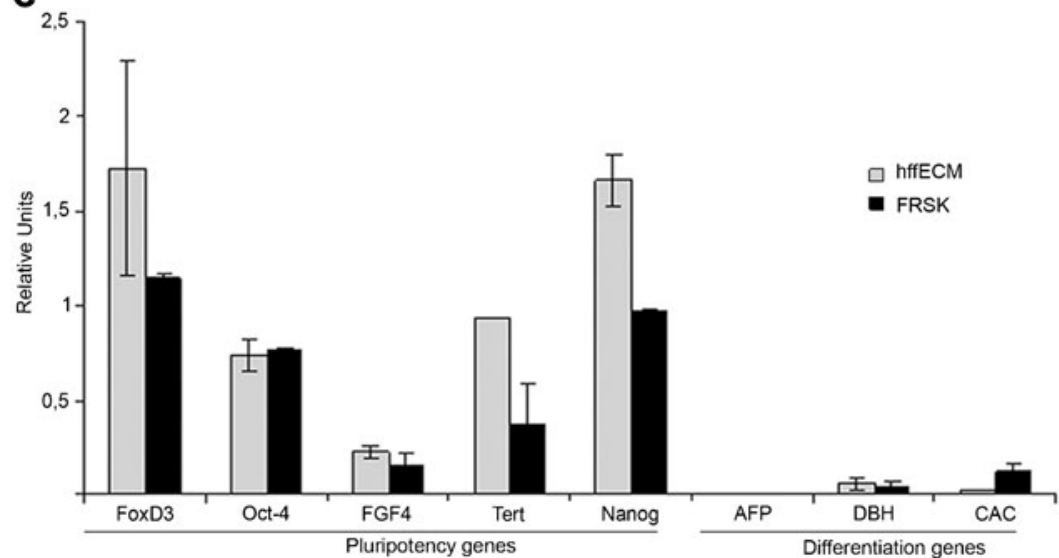

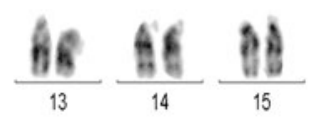

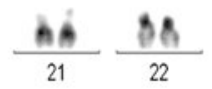

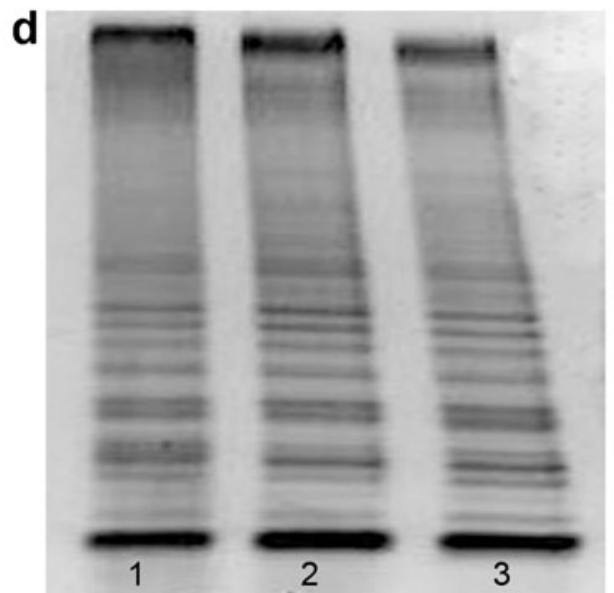

2

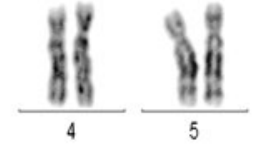

1
Fig. 4 Analysis of pluripotency in cultures of H9 maintained on hffECM. Morphology of H9 colonies maintained for 21 passages on either human foreskin fibroblasts a or hffECM $\mathbf{b}$. Immunodetection of the undifferentiated marker Tra1-60 in H9 colonies maintained during 21 passages on foreskin fibroblasts (a2), or after 21 passages on hffECM (b2). Nuclei were stained with DAPI (blue) and Alexa 488 secondary antibodies (green) were used to detect anti-Tra1-60. All the images were captured at 10X magnification in a Zeiss Axiovert $200 \mathrm{M}$ fluorescence microscope. c Kariotype analysis. After 31 passages on hffECM, hESC cells conserve normal 46XX kariotype. d Analysis of telomerase activity in $\mathrm{H} 9$ cells (passage $31+21$ ). Telomerase activity of $\mathrm{H} 9$ cells at passage 31 before the onset of experiments (lane 1) and colonies maintained on hffECM for 21 additional passages with conditioned media (lane 2) or on foreskin fibroblasts (lane 3). e Quantitative PCR analysis of pluripotent gene expression in H9 cultures maintained for 21 passages on hffECM or foreskin fibroblasts surface and reduce the bioactivity of the surface itself. Disruption of the collagen network changes the mechanical properties and collagen fiber kinetics of the scaffold, which can alter the environment to which cells are exposed [30]. Given these considerations, we compared the capacity of our hffECM to support cell migration events with standard materials such as Matrigel and plastic. A migration assay was performed using the human brain tumor cell line U87, routinely used for migration studies [31], which was plated on the three different supports. Subsequently, we recorded the trajectory and direction changes of at least 20 cells migrating on each of the supports. Cells plated on plastic 

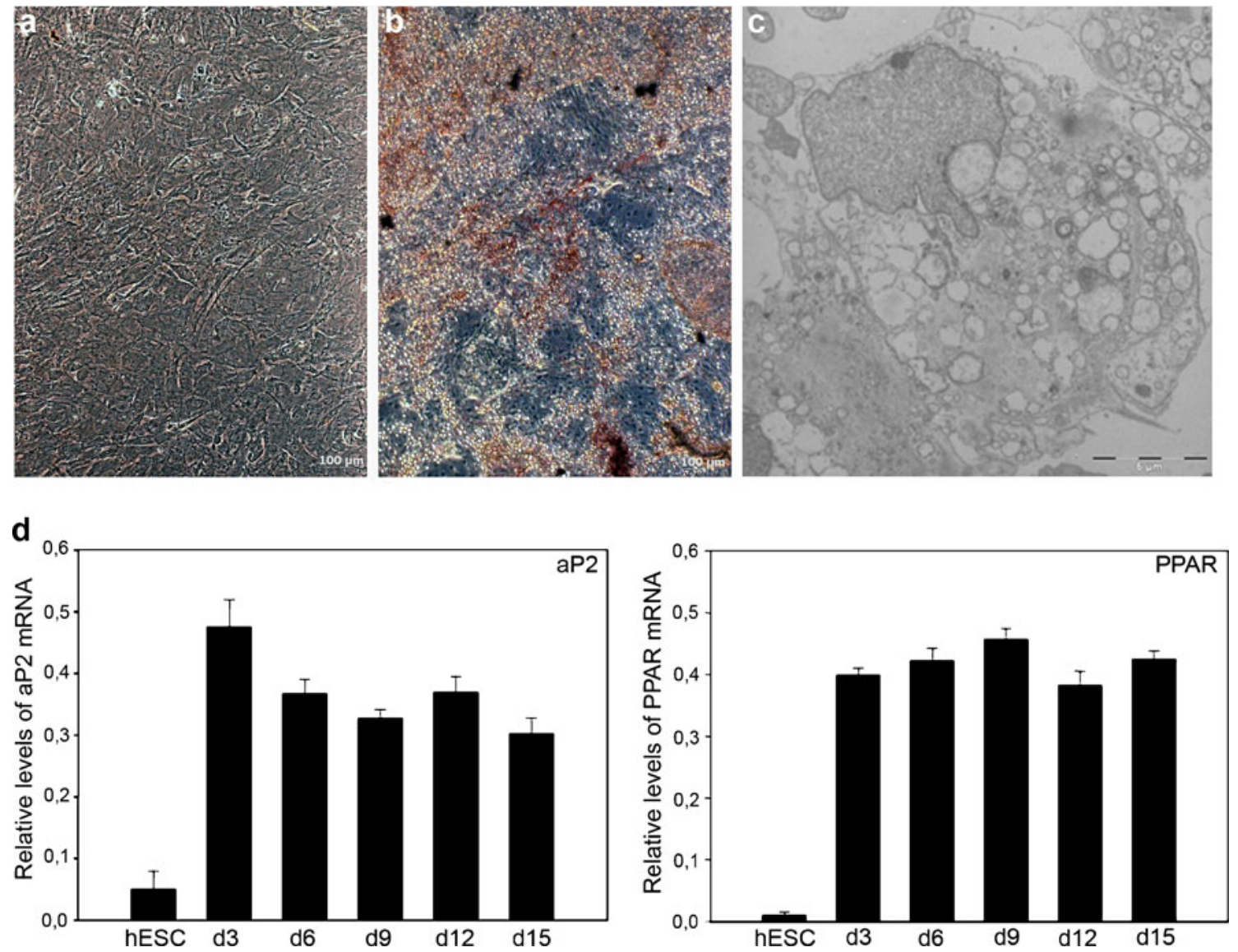

Fig. 5 Evaluation of hffECM as a support for directed differentiation of hESC. Phase contrast images of hESC plated on hffECM and subjected to a chemically-defined method for differentiation to adipocytes. a 12 days in differentiation media, b Oil red $\mathrm{O}$ staining of H9 cultures on day 11 of differentiation protocol. Scale bars: $100 \mu \mathrm{m}$, c Transmission electron microscopy characterization of adipocytes obtained from H9 after 15 days of the differentiation

(Fig. 6a) changed continuously their trajectory and direction as they moved around the center of the well. In contrast, the cells maintained on either Matrigel or hffECM migrated longer distances inside the well. Additionally, the movement of cells on these supports was less haphazard and direction changes were reduced drastically. Regarding the speed of migration, cells grown on hffECM moved significantly faster while no differences were observed between Matrigel and plastic (Fig. 6b). Representative migration patterns of the U87 cells cultured on the various supports can be viewed in the videos $1-3$ of Supplementary Information.

\section{Discussion}

The development of a standardized, animal-free culture matrix is an essential tool for both elucidating the mechanisms that control human stem cell biology and for protocol. The cells display small nuclei, with sparse organelles and large lipid droplets distributed along the cytoplasm. Scale bar: $5 \mu \mathrm{m}$. d Analysis of adipocyte differentiation markers using RT-PCR. At the indicated time points, mRNA was prepared for analysis by PCR. Products were visualized by gel electrophoresis. The expression of aP2 and PPAR gamma was quantified and normalized to $18 \mathrm{~s}$ mRNA

facilitating their application to cell-based clinical therapies [32]. Here we report an efficient method for the obtention and preservation of a functional ECM from human foreskin fibroblasts. Our method has several key advantages over other strategies for obtaining ECM. First of all, it is a simple, low-cost biochemical method based on hypotonic lysis that can be reproduced easily in cell biology labs. Additionally, in contrast to previous chemical procedures that disrupt some of the ECM protein structures [29, 33], the hypotonic shock used in our method preserves the architecture and bioactivity of the ECM scaffold. Indeed, our hffECM was capable of maintaining hESC in the undifferentiated state without inducing chromosomal aberrations during a significant amount of time (21 passages). One observation regarding the hffECM that we initially interpreted as a limitation was the slight reduction in the number of colonies when hESC were continuously passaged on hffECM as compared with those grown on HFF. 


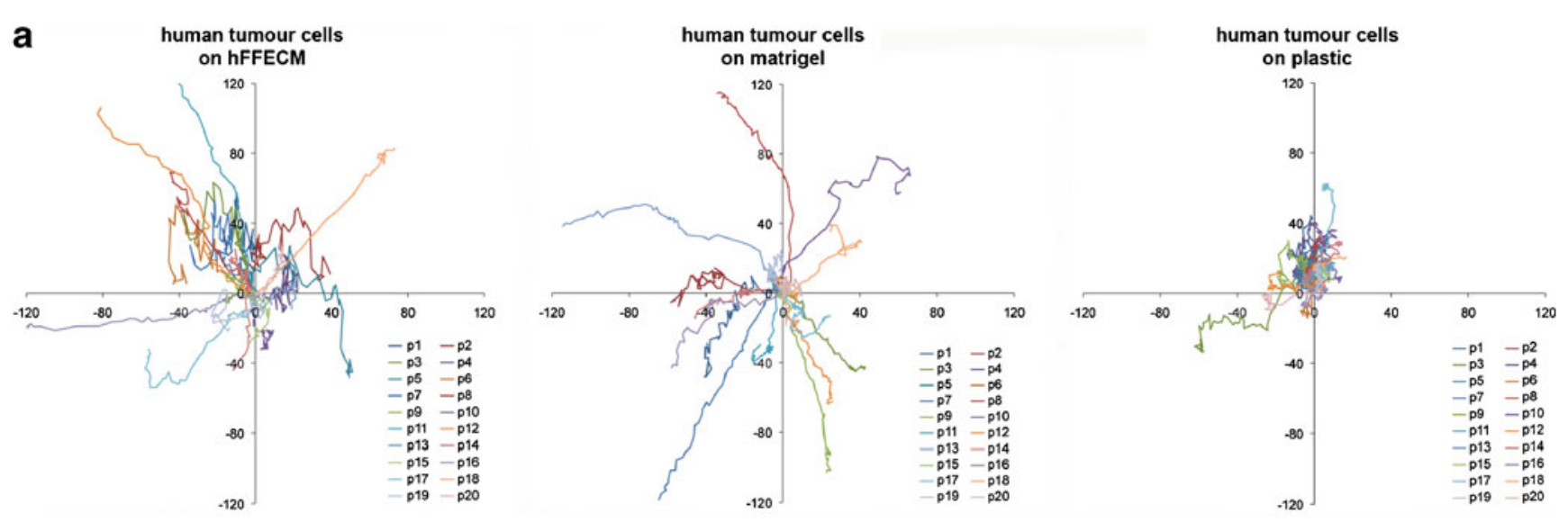

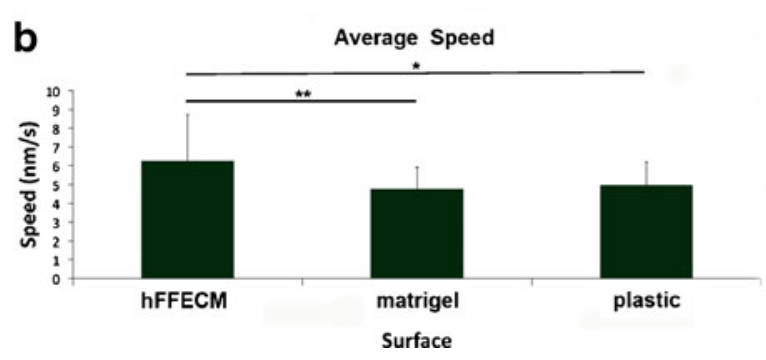

Fig. 6 Analysis of random migration of human tumour cells on hffECM, Matrigel and plastic. a Plots representing the different trajectories followed by 20 randomly chosen cells growing on
hffECM, Matrigel and plastic. b Histogram depicting the average speed of cells growing on the three different surfaces. ${ }^{*} P<0.00007$; ** $P<0.0008$
However, when we analysed markers of pluripotency, the expression of certain pluripotent genes such as Nanog and FoxD3 was enhanced in the colonies maintained on hffECM relative to the colonies on HFF, suggesting that, although the attachment of hESC may be lower on hffECM than on feeders, cells display a higher grade of pluripotency when grown on this support.

Proteomic studies confirmed that the majority of proteins which comprise the ECM $[34,35]$ were present in hffECM after the decellularization induced by osmotic shock of HFF, demonstrating that the procedure prevents enzymatic degradation of the ECM. To date, proteomics has not been applied to define the ECM required for the maintenance and differentiation of hESC. The attachment and growth of all types of cells is dependent on the interaction of integrins expressed on the cell surface with the ECM. The subset of integrins expressed in undifferentiated hESC is modified during differentiation, implicating an important role for integrins in maintaining pluripotency and growth [36]. Our proteomics data reveal that the majority of the ECM partners which interact with integrins are retained in the hffECM (Fig. 3b), providing at least one molecular explanation for the capacity of this matrix to support growth and pluripotency of hESC during an extended period of time (21 passages). Recent studies have demonstrated that both integrin signaling and E-Cadherin signaling are required for the survival and proliferation of $\mathrm{hESC}$ [13]; enzymatic dissociation of hESC colonies induces apoptosis by disrupting these critical cell-ECM interactions. Collectively, our results demonstrate that hffECM retains the composition and integrity of ECM molecules which modulate self-renewal and survival of hESC. Thus, the use of mass spectrometry techniques to precisely define the protein composition of the hffECM may enable the synthesis of a functional support on a large-scale and provides basic information to tailor the composition to particular cell types.

When hESC on hffECM were subjected to differentiation using chemically-defined conditions, we observed satisfactory results. Using an established protocol [27], the efficiency of differentiation to adipocytes was comparable to colonies maintained initially on feeder layers. Combinations of growth factors and ECM can mimic in vitro the cell-cell interactions that occur during development, but data concerning the functional or spatial similarities with real embryo development are lacking [15]. Thus, hffECM provides an excellent opportunity to differentiate hESC towards the three embryonic germ 
layers on a complex and basement-rich support where the ' basal lamina in vivo.

During embryo development or tumour progression, cells usually migrate within a 3D extracellular matrix environment. The majority of in vitro assays have been performed using $2 \mathrm{D}$ models. Computational studies have predicted that some crucial migration parameters such as matrix stiffness and the distribution of integrin forces at cell matrix-interface are dramatically different in $2 \mathrm{D}$ versus $3 \mathrm{D}$ environment [37]. With the aim of extrapolating in vitro experiments to in vivo processes, Zaman et al., 2006 [38] compared cell migration parameters in 2D [39] with Matrigel and observed clear differences between these supports. When we compared the migration of U87 cells on Matrigel versus hffECM as 3D supports, we observed similar patterns, although the speed of migration was superior on hffECM.

In summary, we have developed a simple method that generates a biologically active human ECM under conditions which are compatible with clinical-grade studies. Our hffECM supports the maintenance and differentiation of hESC and based on our data, can be applied to other types of stem cells which require a physical support.

\section{Materials and Methods}

\section{Culture and Maintenance of hESC}

The present study was approved by the Commission for Control of Transplants of the Carlos III Health Institute and complies with the Spanish regulations for hESC research. The human embryonic stem cell lines H9 and H1 were purchased from Wicell (Wisconsin, USA) and cultured as previously described [16]. H9 and H1 colonies were routinely passaged mechanically and maintained on human foreskin fibroblasts (HFF) at 5\% $\mathrm{CO}_{2}$ and $37^{\circ} \mathrm{C}$ until the onset of the experiments described below.

\section{Culture of Human Fibroblasts and Preparation of Conditioned Media}

Human foreskin fibroblasts (ATCC Catalog No. CRL-2429, Passage 11-18) were grown in Dulbecco's modified Eagle medium (DMEM) with high glucose, supplemented with $10 \%$ human serum (HS), 1\% L-glutamine, and $1 \%$ nonessential amino acid (NEAA). The HFF were split using Triple Select every 5-7 days (all the above reagents are from GIBCO, Invitrogen). When the cells reached confluence, they were inactivated by $10 \mu \mathrm{g} / \mathrm{ml}$ Mitomycin C (Fluka, Catalog No. 69824) for 3 h, then washed three times with PBS, digested, and $6 \times 10^{6}$ cells were seeded in
T75 flask coated with $0.1 \%$ gelatin (Sigma, Catalog No. G1890). To generate conditioned media, cells were cultured at $37^{\circ} \mathrm{C}, 5 \% \mathrm{CO} 2$ in one of the following: Medium 1 or TESR medium. Medium 1 contained $80 \%$ KnockoutDulbecco's modified Eagle's medium (KO-DMEM), 20\% KNOCKOUT serum replacement (Knockout SR), 1\% L-glutamine, 1\% NEAA, 100 $\mathrm{MM}$ 2-Mercaptoethanol (Sigma, Catalog No.M7522). Medium was collected daily during 1 week of culture. Before use as the culture medium for hESC, $4 \mathrm{ng} / \mathrm{ml}$ human basic fibroblast growth factor (bFGF) (Invitrogen, Catalog 13256-029) was added. Conditioned medium was stored at $-80^{\circ} \mathrm{C}$ for up to 6 months with no loss of biological activity.

\section{Preparation of ECM from HFF(hffECM)}

Extracellular matrix was prepared by buffer extraction of human foreskin fibroblasts, using methods described by Cukierman et al. 2001 [40] and Hedman et al. 1979 [41] with some modifications. HFF cultures were washed twice with PBS and treated with lysis buffer $(10 \mathrm{mM}$ Tris, $1 \mathrm{mM}$ EDTA, pH 7.4) using $1.5 \mathrm{ml}$ per $10^{6}$ cells. The cells were incubated overnight at $4^{\circ} \mathrm{C}$ on an orbital shaker. Lysed cells were eliminated from the plates by washing six times with PBS. Plates were stored at $4^{\circ} \mathrm{C}$ or dried and sterilized using standard methods.

\section{Elution of hffECM}

After preparation of the hffECM as described above, culture plates were treated with $10 \mathrm{mM}$ DTT in $5 \mathrm{M}$ sodium guanidine for $1 \mathrm{~h}$ at $4{ }^{\circ} \mathrm{C}$. The solubilized matrix was collected by scraping the culture plates, followed by three washes with the solubilization buffer. Samples were then centrifuged for $20 \mathrm{~min}$ at high speed to concentrate the eluted protein. Using these conditions, laminin was not detected in the initial proteomic analysis. However, laminin was noted as a component of the hffECM by IHC performed on culture plates (Fig. 2). Thus, we used the extraction protocol described by Kleinman et al. [42] with slight modifications because it preserves the native structure of the protein. Culture plates were incubated in $3.4 \mathrm{M}$ $\mathrm{NaCl}$ buffer for $1 \mathrm{~h}$ at room temperature. Eluted samples were then centrifuged $5 \mathrm{~min}$ at $8000 \times \mathrm{g}$ at $4^{\circ} \mathrm{C}$. The supernatants were discarded and after the addition of $0.5 \mathrm{M} \mathrm{NaCl}$ buffer to the pellets, they were solubilized overnight at $4^{\circ} \mathrm{C}$. Subsequently, the homogenate was centrifuged $15 \mathrm{~min}$ at $8000 \times \mathrm{g}$ at $4^{\circ} \mathrm{C}$. The resulting supernatant was collected on ice and ammonium sulfate was added to $30 \%$ saturation $(16.4 \mathrm{~g} / 100 \mathrm{ml})$ with vigorous stirring. Finally, these samples were centrifuged $15 \mathrm{~min}$ at $8000 \times g$ at $4^{\circ} \mathrm{C}$ and the pellet was recovered and resuspended [42]. 
Proteomic Analysis of hffECM Protein Components

The hffECM samples were analyzed in two different ways. Samples from both protocols were digested in solution with sequencing grade trypsin (Promega) following the protocol described by Shevchenko et al. [43]. In addition, the ECM sample obtained by the second extraction procedure was analyzed by SDS/PAGE using Bio-Rad Mini-PROTEAN TGX precast gels with a $4-15 \%$ resolving gel. The gel lane was cut into pieces, digested [44], and analyzed separately. The digestion mixture was dried in a vacuum centrifuge, resuspended in $6 \mu \mathrm{L}$ of $0.1 \%$ TFA, and $5 \mu \mathrm{L}$ were delivered to a trap column (LC Packings Amsterdam, The Netherlands, C18 PepMap100, $5 \mu \mathrm{m}, 300 \mu \mathrm{m} \times 5 \mathrm{~mm}$ ) using a capillary HPLC (Switchos, LC Packings) via an isocratic flow of mobile phase $(0.1 \%$ trifluoroacetic acid in water) at a flow rate of $30 \mu \mathrm{L} / \mathrm{min}$ for $3 \mathrm{~min}$. The flow rate was the switched to $200 \mathrm{~nL} / \mathrm{min}$, and the peptides were flushed onto the analytical column (LC Packings C18 PepMap100, $3 \mu$ $100 \AA, 75 \mu \mathrm{m} \times 15 \mathrm{~cm}$ ) and eluted via a mobile phase linear gradient: $15-50 \%$ B (A: $0.1 \%$ formic acid in water, B: $0.1 \%$ formic acid in $95 \%$ acetonitrile) over $150 \mathrm{~min}$. The eluted peptides were directly infused in a QSTAR XL mass spectrometer (Applied Biosystems). The nanoESI QTOF hybrid system was operated in information-dependent acquisition mode, in which a 1-s TOF MS scan from 400 to $2000 \mathrm{~m} / \mathrm{z}$, was performed, followed by 1 -s product ion scans from 100 to $2000 \mathrm{~m} / \mathrm{z}$ on the three most intense doubly or triply charged ions. The MS/MS information was sent to MASCOT $\mathrm{v}$ 2.2. using the MASCOT DAEMON software $\mathrm{v}$ 2.2.2.

Protein Database Search and Functional Annotation

Peak list generation was performed with Mascot Daemon 2.2.2 (Matrix Science, Inc., Massachusetts, USA) using Mascot.dll 1.6b25 or with ProteinPilot 3.0 (ABSCIEX) using ABSciex. DataAccess.Wiff File DataReader.dll. Mascot 2.2 and ProteinPilot 3.0 were used to search Uniprot and NCBInr databases. Mascot search parameters set trypsin cleavage specificity, allowing one mis-cleavage, with mass tolerances of $100 \mathrm{ppm}$ and 0.6 Da for MS and MS/MS modes, respectively. Cysteine carbamidomethylation as fixed modification and methionine oxidation as well as glutamine and asparagine deamidation as variable modifications were specified. The taxonomy was set to Homo sapiens. The Paragon algorithm used by ProteinPilot does not require the manual setting of any of these parameters.

Functional annotation was performed with the FatiGO+ module of Babelomics to explore enrichment of gene ontology terms (GO) and KEGG pathways within the identified proteins $[25,26]$. The significant level was set to a $p$-value of 0.01 . We focused on the Cellular Compartment terms of GO in order to evaluate the precedence of the identified proteins. Interactions among the proteins belonging to the significantly enriched cellular components were analyzed with STRING. Clustering of the networks was done using the MCL algorithm with the parameter "inflation" set to 2 .

\section{Ultrastructural Study of hffECM Organization} and Thickness

For ultrastructural analysis of ECM organization, ECM coated plates as well as the corresponding controls containing feeder cells, were repeatedly washed in a 0,1 M phosphate buffer ( $\mathrm{PB}$; pH 7.4) solution, before fixation for Transmission Electron Microscopy (TEM). Fixation was performed in 3\% glutaraldehyde solution in $\mathrm{PB}$ for $30 \mathrm{~min}$ at $37^{\circ} \mathrm{C}$ and postfixed in $2 \% \mathrm{OsO}_{4}$ in PBS. Samples were stained with $2 \%$ uranyl acetate (Electron Mycroscopy Science, Washington). Dehydration was achieved by a graded series of ethanol and samples were embedded in propylene oxide (Lab Baker, Deventry, Holland). Finally, plates were incubated with araldite (Durkupan, Fluka) overnight.Following polymerization, embedded samples were detached from the chamber slide and glued to Araldite blocks. Serial semi-thin $(1.5 \mu \mathrm{m})$ sections were cut with an Ultracut UC-6 (Leica, Heidelberg, Germany) and mounted onto slides and stained with $1 \%$ toluidine blue. Selected semi-thin sections were glued (Super Glue, Loctite) to araldite blocks and detached from the glass slide by repeated freezing (in liquid nitrogen) and thawing. Ultrathin $(0.07 \mu \mathrm{m})$ sections were prepared with the Ultracut and stained with lead citrate. Finally, photomicrographs were obtained under a transmission electron microscope (FEI Tecnai Spirit G2) using a digital camera (Morada, Soft Imaging System, Olympus).

For immunocytochemistry, the plates containing hffECM were fixed in $2 \%$ paraformaldehyde and washed three times with PBS before staining o.n. with mouse antihuman fibronectin (1:250 Sigma), rabbit anti-human collagen type I (1:400 Millipore), mouse anti-human laminin (1:500 Sigma). Non-specific binding was blocked with normal goat serum in PBS. For secondary detection depending on the primary, goat anti-mouse Alexa 488 and goat anti-rabbit Alexa 647 were used (1:500 Millipore). Following incubation with secondary antibodies, cells were washed four times with PBS and mounted using Prolong Gold AntiFade Reagent with Dapi (Invitrogen). Images were obtained using the Leica SP2 confocal.

\section{Analysis of Pluripotency}

For immunocytochemistry, culture media was removed from the hESC and cells were washed carefully with 
PBS. Cells were fixed 30 min with $4 \%$ paraformaldehyde. Subsequently, cells were washed three times with PBS and permeabilized with $0.5 \%$ Triton X-100 (Sigma- Aldrich) at room temperature. Non-specific binding was blocked with normal goat serum in PBS. Primary antibodies (SSEA-3/4, SSEA-1, TRA- 1-60, TRA-1- 81) of the ES Cell Characterization Kit (1:50 Millipore) were diluted according to the manufacturer's instructions and incubated overnight at $4^{\circ} \mathrm{C}$. For detection with secondary antibodies, IgG1 Alexa488 was used for SSEA-4, and IgM Alexa488 for Tra1-60 and Tra1-80. After $1 \mathrm{~h}$ of incubation at room temperature, samples were washed four times with PBS and mounted using Prolong Gold Anti-Fade Reagent with Dapi (Invitrogen). Images were obtained at a $10 \mathrm{X}$ magnification using the Zeiss Axiovert $200 \mathrm{M}$.

\section{Chromosomal Analysis}

Cytogenetic analysis was conducted at passages 17 and 21 after transfer of hESC colonies to hffECM-coated plates. Dividing cells were arrested at metaphase by the addition of $50 \mu \mathrm{g} / \mathrm{ml}$ of colchicine for $2 \mathrm{~h}$ and then harvested using standard cytogenetic techniques. Harvested metaphase chromosomes were stained. Giemsa/Trypsin/Leishman (GTL) banding techniques were employed for karyotype analysis. An average of 30 banded metaphase spreads were karyotyped and analyzed for chromosomal rearrangements. GTL- banded metaphase spreads were captured and arranged using Cytovision software from Applied Imaging (Santa Carla, CA, USA).

\section{RNA Preparation and RT-PCR}

Total RNA was prepared from cells using RNeasy mini kit (Quiagen, Gilden; no. 74104). To eliminate contaminating genomic DNA, the initial RNA pellet was incubated with deoxyribonuclease (DNase) I (2 to $4 \mathrm{U} / \mu \mathrm{L}$; Qiagen, Carlsbad; no.79254) for $15 \mathrm{~min}$ at room temperature in the buffer supplied by the manufacturer. RT-PCR and primer sequences were as described in the Supplemental Table S2. They were designed using Primer3 software and synthesized by Sigma-Aldrich. For each experiment, controls were performed in which reverse transcriptase was omitted from the cDNA reaction mixture and template DNA was omitted from the PCR mixture. For quantitative real-time PCR (QRT-PCR), $5 \mu \mathrm{g}$ RNA was converted into cDNA, and a series of diluted samples were used for 40-cycle PCR in Light Cycler 480 SYBR Green I Master (Kit no. 04707516001) in an Lightcycler 480 (Roche Diagnostics,Mannheim) instrument. Reactions (20 $\mu \mathrm{L}$ total) contained $1 \mu \mathrm{L}$ cDNA, $10 \mu \mathrm{M}$ each primer, and $4 \mu \mathrm{M}$ probe and were run using the default Lightcycler 480 program. To generate a standard curve for comparison of mRNA levels in different samples, multiple dilutions of the control cDNA sample, spanning at least 3 orders of magnitude, were prepared. The equation describing the plot of threshold cycle, $C_{t}$, versus $\log$ concentration was used to determine relative amounts of mRNA in experimental samples. Using the optimized conditions and threshold values, individual samples were analyzed in triplicate using the probe of interest and an internal control expected to be unchanged between samples. Three different internal controls were used: glyceraldehyde-3-phosphatedehydrogenase (Gapdh), $\beta-2$ microglobulin, and $\beta$-actin. From the $C_{t}$ values, the relative transcript concentration was calculated and normalized to that of the internal control. The maximum expression data point was adjusted to 100 . Data are shown for samples normalized to Gapdh, but results were comparable when analysis was performed using either $\beta-2$ microglobulin alone or a combination of all 3 controls.

\section{Telomerase Activity Assay}

Telomerase activity was assayed by telomere repeat amplification protocol using Trapeze Kit (Chemicon) and according to the manufacturer's protocol.

\section{Analysis of hESC Differentiation}

Spontaneous in vitro differentiation was evaluated by allowing hESC to overgrow on hffECM in growth media without basic fibroblast growth factor bFGF (Invitrogen). To induce adipocyte differentiation, the hESC were seeded in triplicate on ECM-coated wells of a 6-well plate at a density of around $30 \mathrm{hESC}$ colonies/well (about 300-500 cells per colony) in CM. Cultures were then maintained continuously in Differentiation medium 1 (DM1): 80\% Knock-out Dulbecco's modified Eagle's medium DMEM/F12, 20\% Knock-out serum replacement, $1 \mathrm{mmol} / \mathrm{L}$ L-glutamine, $100 \mu \mathrm{M}$ 2-Mercaptoethanol, 1\% MEM non-essential amino acids. The medium was changed every other day. Culture samples were collected at day 0 , day 3 , day 6 , day 9 , day 12 , and day 15 and analyzed by Oil Red O staining and RT-PCR.

\section{Analysis of Cell Migration}

The human brain tumour cell line U-87 MG was grown in Ham's F-12 supplemented with $10 \%$ FBS. For the assay, $5 \times 10^{3}$ per well were seeded onto three replicate 96-well plates coated with Matrigel, hffECM or plastic respectively, and maintained for at least $3 \mathrm{~h}$ in a incubator before the experiment. During the migration experiment $(8 \mathrm{~h})$, plates were placed in a pre-equilibrated In-Cell Analyzer 1000 Chamber and imaged every 10 min using 
10X objective with 2000 exposure. Images were analyzed with In-Cell Investigator software using the cell tracking analysis tool to create plots of X-Y position and time variables. The particles with the same identification number were connected by a line representing the continuous track for a cell (GE Healthcare application Note 28-9327-11 AA). For statistical comparison of the parametric data obtained, we used the $t$-test analysis of variance for two groups.

\begin{abstract}
Acknowledgements We are grateful to Dr. Jose Manuel Garcia-Verdugo for his assistance with electron microscopy and to Enrique Navarro and $\mathrm{M}^{\mathbf{a}}$ Teresa Minguez of the University of Valencia for technical support with the confocal microscope. Additionally, we thank Dr. Clara Rodríguez and Sandra Pinto for their assistance with the migration assays. Carmen Escobedo-Lucea is financed by post-doctoral program Sara Borrell from Institute of health Carlos III, Ministry of Health, Spain. This study was supported with financial support from the Regenerative Medicine Program of the Valencian Community, Prometeo 2009/011, TerCel, CIBERDEM (ISCIII), the Spanish Ministry of Science and Innovation (SAF2008-00011), EMER07 (ISCIII) and LIVES, an initiative of the FP7 program. The Proteomics core facility of the CIPF is a member of ProteoRed-ISCIII
\end{abstract}

Open Access This article is distributed under the terms of the Creative Commons Attribution Noncommercial License which permits any noncommercial use, distribution, and reproduction in any medium, provided the original author(s) and source are credited.

\section{References}

1. Thomson, J. A., Itskovitz-Eldor, J., Shapiro, S. S., et al. (1998). Embryonic stem cell lines derived from human blastocysts. Science, 282, 1145-1147.

2. Takahashi, K., \& Yamanaka, S. (2006). Induction of pluripotent stem cells from mouse embryonic and adult fibroblast cultures by defined factors. Cell, 126, 663-676.

3. Zuk, P. A., Zhu, M., Mizuno, H., et al. (2001). Multilineage cells from human adipose tissue: implications for cell-based therapies. Tissue Engineering, 7, 211-228.

4. Macchiarini, P., Jungebluth, P., Go, T., et al. (2008). Clinical transplantation of a tissue-engineered airway. Lancet, 372, 2023-2030.

5. Atala, A., Bauer, S. B., Soker, S., Yoo, J. J., \& Retik, A. B. (2006). Tissue-engineered autologous bladders for patients needing cystoplasty. Lancet, 367, 1241-1246.

6. Martin, M. J., Muotri, A., Gage, F., \& Varki, A. (2005). Human embryonic stem cells express an immunogenic nonhuman sialic acid. Natural Medicines, 11, 228-232.

7. Rodin, S., Domogatskaya, A., Strom, S., et al. Long-term selfrenewal of human pluripotent stem cells on human recombinant laminin-511. Nature Biotechnology, 28, 611-615.

8. Spradling, A., Drummond-Barbosa, D., \& Kai, T. (2001). Stem cells find their niche. Nature, 414, 98-104.

9. Kagami, S., Kondo, S., Loster, K., et al. (1998). Collagen type I modulates the platelet-derived growth factor (PDGF) regulation of the growth and expression of betal integrins by rat mesangial cells. Biochemical and Biophysical Research Communications, 252, 728-732.

10. Derda, R., Li, L., Orner, B. P., Lewis, R. L., Thomson, J. A., \& Kiessling, L. L. (2007). Defined substrates for human embryonic stem cell growth identified from surface arrays. ACS Chemical Biology, 2, 347-355.

11. Ludwig, T. E., Bergendahl, V., Levenstein, M. E., Yu, J., Probasco, M. D., \& Thomson, J. A. (2006). Feeder-independent culture of human embryonic stem cells. Nature Methods, 3, 637646.

12. Ludwig, T. E., Levenstein, M. E., Jones, J. M., et al. (2006). Derivation of human embryonic stem cells in defined conditions. Nature Biotechnology, 24, 185-187.

13. Xu, C., Inokuma, M. S., Denham, J., et al. (2001). Feeder-free growth of undifferentiated human embryonic stem cells. Nature Biotechnology, 19, 971-974.

14. Amit, M., Shariki, C., Margulets, V., \& Itskovitz-Eldor, J. (2004). Feeder layer- and serum-free culture of human embryonic stem cells. Biology of Reproduction, 70, 837-845.

15. Klimanskaya, I., Chung, Y., Meisner, L., Johnson, J., West, M. D., \& Lanza, R. (2005). Human embryonic stem cells derived without feeder cells. Lancet, 365, 1636-1641.

16. Stojkovic, P., Lako, M., Przyborski, S., et al. (2005). Human-serum matrix supports undifferentiated growth of human embryonic stem cells. Stem Cells, 23, 895-902.

17. Klim, J. R., Li, L., Wrighton, P. J., Piekarczyk, M. S., \& Kiessling, L. L. A defined glycosaminoglycan-binding substratum for human pluripotent stem cells. Nature Methods, 7, 989-994.

18. Mei, Y., Saha, K., Bogatyrev, S. R., et al. Combinatorial development of biomaterials for clonal growth of human pluripotent stem cells. Nature Materials, 9, 768-778.

19. Hodde, J. P., Record, R. D., Tullius, R. S., \& Badylak, S. F. (2002). Retention of endothelial cell adherence to porcine-derived extracellular matrix after disinfection and sterilization. Tissue Engineering, 8, 225-234.

20. Hodde, J. P., Badylak, S. F., Brightman, A. O., \& Voytik-Harbin, S. L. (1996). Glycosaminoglycan content of small intestinal submucosa: a bioscaffold for tissue replacement. Tissue Engineering, 2, 209-217.

21. Hodde, J. P., Record, R. D., Liang, H. A., \& Badylak, S. F. (2001). Vascular endothelial growth factor in porcine-derived extracellular matrix. Endothelium, 8, 11-24.

22. Badylak, S. F., Freytes, D. O., \& Gilbert, T. W. (2009). Extracellular matrix as a biological scaffold material: structure and function. Acta Biomaterialia, 5, 1-13.

23. Ott, H. C., Matthiesen, T. S., Goh, S. K., et al. (2008). Perfusiondecellularized matrix: using nature's platform to engineer a bioartificial heart. Natural Medicines, 14, 213-221.

24. Escobedo-Lucea, C., \& Stojkovic, M. (2010). Growth of human embryonic stem cells using derivates of human fibroblasts. Methods Mol Biol, 584, 55-69.

25. Al-Shahrour, F., Minguez, P., Tarraga, J., et al. (2006). BABELOMICS: a systems biology perspective in the functional annotation of genome-scale experiments. Nucleic Acids Research, 34, W472-W476.

26. Al-Shahrour, F., Minguez, P., Tarraga, J., et al. (2007). FatiGO +: a functional profiling tool for genomic data. Integration of functional annotation, regulatory motifs and interaction data with microarray experiments. Nucleic Acids Research, 35, W91-W96.

27. Xiong, C., Xie, C. Q., Zhang, L., et al. (2005). Derivation of adipocytes from human embryonic stem cells. Stem Cells and Development, 14, 671-675.

28. D’Amour, K. A., Agulnick, A. D., Eliazer, S., Kelly, O. G., Kroon, E., \& Baetge, E. E. (2005). Efficient differentiation of human embryonic stem cells to definitive endoderm. Nature Biotechnology, 23, 1534-1541.

29. Prado-Lopez, S., Conesa, A., Arminan, A., et al. Hypoxia promotes efficient differentiation of human embryonic stem cells to functional endothelium. Stem Cells, 28, 407-418.

30. Gilbert, T. W., Sellaro, T. L., \& Badylak, S. F. (2006). Decellularization of tissues and organs. Biomaterials, 27, 3675-3683. 
31. Catacuzzeno, L., Aiello, F., Fioretti, B., et al. Serum-activated K and $\mathrm{Cl}$ currents underlay U87-MG glioblastoma cell migration. Journal of Cellular Physiology 2010.

32. Villa-Diaz, L. G., Nandivada, H., Ding, J., et al. Synthetic polymer coatings for long-term growth of human embryonic stem cells. Nature Biotechnology, 28, 581-583.

33. Grauss, R. W., Hazekamp, M. G., Oppenhuizen, F., van Munsteren, C. J., Gittenberger-de Groot, A. C., \& DeRuiter, M. C. (2005). Histological evaluation of decellularised porcine aortic valves: matrix changes due to different decellularisation methods. European Journal of Cardiothoracic Surgery, 27, 566-571.

34. Tong, W. Y., Liang, Y. M., Tam, V., et al. (2010). Biochemical characterization of the cell-biomaterial interface by quantitative proteomics. Molecular \& Cellular Proteomics, 9, 2089-2098.

35. Xu, Y., Zhu, X., Hahm, H. S., et al. Revealing a core signaling regulatory mechanism for pluripotent stem cell survival and selfrenewal by small molecules. Proceedings of the National Academy of Sciences of the United States of America, 107, 8129-8134.

36. Rowland, T. J., Miller, L. M., Blaschke, A. J., et al. (2010). Roles of integrins in human induced pluripotent stem cell growth on Matrigel and vitronectin. Stem Cells and Development, 19, 12311240.

37. Zaman, M. H., Kamm, R. D., Matsudaira, P., \& Lauffenburger, D. A. (2005). Computational model for cell migration in three-dimensional matrices. Biophysical Journal, 89, 13891397.
38. Zaman, M. H., Trapani, L. M., Sieminski, A. L., et al. (2006). Migration of tumor cells in 3D matrices is governed by matrix stiffness along with cell-matrix adhesion and proteolysis. Proceedings of the National Academy of Sciences of the United States of America, 103, 10889-10894.

39. Palecek, S. P., Loftus, J. C., Ginsberg, M. H., Lauffenburger, D. A., \& Horwitz, A. F. (1997). Integrin-ligand binding properties govern cell migration speed through cell-substratum adhesiveness. Nature, 385, 537-540.

40. Cukierman, E., Pankov, R., Stevens, D. R., \& Yamada, K. M. (2001). Taking cell-matrix adhesions to the third dimension. Science, 294, 1708-1712.

41. Hedman, K., Kurkinen, M., Alitalo, K., Vaheri, A., Johansson, S., \& Hook, M. (1979). Isolation of the pericellular matrix of human fibroblast cultures. The Journal of Cell Biology, 81, 83-91.

42. Kleinman, H. K. (2001). Preparation of basement membrane components from EHS tumors. Current Protocol in Cell Biology Chapter 10:Unit 102.

43. Shevchenko, A., Jensen, O. N., Podtelejnikov, A. V., et al. (1996). Linking genome and proteome by mass spectrometry: large-scale identification of yeast proteins from two dimensional gels. Proceedings of the National Academy of Sciences of the United States of America, 93, 14440-14445.

44. Shevchenko, A., Wilm, M., Vorm, O., \& Mann, M. (1996). Mass spectrometric sequencing of proteins silver-stained polyacrylamide gels. Analytical Chemistry, 68, 850-858. 